\title{
Result of Internal Fixation of Femoral Shaft Nonunion with Implant Failure by SIGN Interlocking Nail at Rajshahi Medical College Hospital
}

\author{
Mohd. Alamgir Hossain ${ }^{1}$, Mosammat Nargis Shamima ${ }^{2}$, Ahmed Asif Iqbal ${ }^{3}$ \\ Md. Enamul Haque ${ }^{4}$, Md. Qumruzzaman Parvez ${ }^{5}$, Md. Ahsanuzzaman ${ }^{6}$
}

\begin{abstract}
This prospective study on result of internal fixation of femoral shaft nonunion with implant failure by SIGN interlocking nail was carried out during the period of January 2009 to December 2016at Rajshahi Medical College Hospital, Bangladesh. Mean age of the patient was 34 years (11-70 years), majority of the patients were male (76\%), injury was due to high energy trauma $(88 \%)$, right side involvement was more (68\%).Closed exchange nailing was done in 28 cases (56\%) and open nailing was done in 22 cases (44\%). In the open cases previous implants were removed, refreshening of the fracture ends was done. Antegrade or retrograde SIGN interlocking nailing depend on the site involvement. Reaming materials were used as internal bone graft in all the cases. Autogenouscancellous bone graft was given in 5 cases due to comminution at fracture site ( 2 cases) and severe osteoporosis ( 3 cases). The final outcome satisfactory result was found in $44(88 \%)$ cases and unsatisfactory result in $6(12 \%)$ cases.
\end{abstract}

Key Words: Nonunion, Implant failure, interlocking nailing, bone graft.

TAJ 2017; 30: No-1: 11-16

\section{Introduction}

In spite of increased understanding of biomechanics and implant design, nonunion of femoral shaft fractures continues to hinder the treatment of theseinjuries. ${ }^{1}$ Femoral nonunion is a functional and echonomical challenge for the patient as well as treatment dilema for the orthopaedic surgeon. Femoral shaft fractures may fail to unite because of the severity of the injury, damage to the surrounding soft tissues, inadequate initial fixation and demographic characteristics of the patient. Most of the discussion on long bone nonunion is centered on tibial fractures. ${ }^{1}$ The accepted standard treatment for femoral shaft fracture non-union includes removal of previous intramedullary nail, reaming the intramedullary canal and inserting a larger diameter intramedullary interlocking nail in close method. ${ }^{2-4}$

Openautogenous bone grafting in addition to intramedullary reaming has been recommended only in cases of pseudoarthrosis or non-union with bony defects at the fracture site. ${ }^{1}$

The advent of improved implant design has helped achieve excellent results treating femoral nonunions. ${ }^{5}$ Intramedullary SIGN interlocking fixation in implant failure with non-union of femoral fracture is an effective method of treatment. These devices obtain biological fixation

${ }^{1}$ Assistant Professor, Department of Orthopaedic Surgery, Rajshahi Medical College.

${ }_{2}^{2}$ Assistant Professor, Department of Obs \& Gynae, Rajshahi Medical College.

${ }^{3}$ Assistant Professor, Department of Orthopaedic Surgery, Rajshahi Medical College.

${ }^{4}$ Assistant Professor, Department of Orthopaedic Surgery, Rajshahi Medical College.

${ }^{5}$ Medical Officer, Department of Orthopaedic Surgery, Rajshahi Medical College.

${ }^{6}$ Assistant Registrar, Department of Orthopaedic Surgery, Rajshahi Medical College. 
because they are load sharing rather than load sparing implants and successfully controlling the axial and rotational deforming forces. They are solid nail, stout and prevent infections. The antegrade femoral nailing allows better control of proximal and midshaft fracture, while retrograde femoral nailing is reliable in controlling distal shaft fractures. Wider proximal portion of the nail occupy proximal and distal capacious areas in case of antegrade and retrograde inserted nails respectively and resist angulation and rotation. ${ }^{6}$

In Rajshahi Medical College Hospital we are doing SIGN interlocking nailing both antegrade and retrograde for fixation of femoral shaft fracture nonunion with implant failure. This study is to report the outcome of SIGN interlocking nailing for fixation of femoral shaft fracture nonunion with implant failure will be evaluated.

\section{Materials and Methods}

Between January 2009 to December 2016, 52 patients with nonunion of the shaft of femur fracture with implant failure were evaluated and cared for at Orthopaedic Surgery department of RajshahiMedical College Hospital.

For the purposes of the study, non-union is defined as absence of clinical or radiological healing 9 months after fracture. The shaft of femur is defined as the area $5 \mathrm{~cm}$ distal to the lesser trochanter proximally and $5 \mathrm{~cm}$ proximal to epicondylar axis distally.

This prospective study was undertaken to evaluate the results of fixation of femoral shaft nonunion with implant failure by SIGN interlocking intramedullary nail.

Purposive shampling method was followed as per inclusion and exclusion criteria. Selection was done on the basis of history, clinical examination and radiological evaluation at the SIGN follow up room of Orthopaedic Surgery department of Rajshahi Medical College Hospital.

A complete history of the selected cases was taken with particular emphasis to the time and mechanism of injury, previous treatment and was assessed to rule out any co-existing diseases (Diabetes mellitus, Hypertension, Collagen tissue disorder etc.) This was followed by a through general and physical examination to exclude any associated injuries. Available X-rays AP and lateral views were evaluated to determine type of nonunion (Hypertropic or atropic), fracture alignment, fracture configuration, status of previous implants and status of union.

Preoperatively lateral X-ray view of the patient's femur was done to estimate the length and diameter of the required SIGN nail. The diameter of the nail was estimated by measuring the diameter of intramedullary canal at the narrowest point. Magnification was subsided 10 percent. Finally exact nail length and diameter was determined during surgery.

Antegrade nailing for proximal and middle third fracture and retrograde nailing for distal third fracture was done. All the 52 patients were operated under spinal anaesthesia. Two gram ceftriaxone was given in intravenous route routinely in all the cases.

During operation patients were placed in the lateral position with the affected side uppermost for antegrade nailing and patients were placed in the supine position with flexion of the knee at least 60 degree for retrograde nailing. In patients with intact intramedullary nail, which was removed, over reaming was done in the hope of endosteal bone regeneration as well as fixation stability by putting bigger diameter nails. In patients with broken intramedullary nail or Plates, previous implants were removed, refreshening of the fracture ends and intramedullary canal was reamed in $1 \mathrm{~mm}$ increments till chattering. After reduction by manual traction or hinge and maintaining rotational alignment, reduced fracture ends were held in position by bone holding forceps or lowman's clump.

For antegrade nailing about $5 \mathrm{~cm}$ incision was made from trochanteric flare obliquely upwards in the line with the fibres of the gluteal maximus. For retrograde nailing a midline incision was made over the patellar tendon.

Using a curved femoral awl the entry portal for antegrade nailing was made over the lateral aspect of tip of greater trochanter at the junction of anterior $2 / 3^{\text {rd }}$ and posterior $1 / 3^{\text {rd }}$ and for retrograde 
nailing entry portal was made through the medial side of the nonarticular part of the intercondylar area just anterior to the femoral attachment of the posterior cruciate ligament.

After the creation of entry portal SIGN pilot reamer was introduced and we gradually increased the diameter of the reamer till chattering. Few portion of the entry side was over reamed for accommodating proximal bending of the nails.

Then the desired nail was fixed at SIGN jig and checked. Then the nail was advanced across the fracture site keeping the fracture ends in proper alignment to avoid splintering.

After the fracture was fixed by the SIGN nail and interlocking screws, the jig from the L-handle and L-handle from the nail were removed. Irrigation of the wound was done with normal saline and the wound were closed in layers keeping a drain in situ.

Postoperatively the operated limb was elevated on a pillow keeping the knee in slight flexion. The patients started isometric quadriceps exercises 24 hours after operation. After 48 hours, drain was removed. The patients were allowed to move out of the bed when pain permitted, using crutches with partial weight bearing on operated limb and advised knee and hip bending exercise. Stitches were removed on $14^{\text {th }}$ postoperative day. Postoperatively antibiotics were given routinely for two weeks in all cases. Patients were discharged with the advice to move the hip and knee joints actively from third week.

All patients were evaluated both clinically and radiologically at $1,3,6,12,18$ and 24 months. If there was radiological evidence of healing (callus), the patient was allowed to full weight bearing with crutches. After seeing radiological evidence of consolidation on check X-ray the patient was advised to leave the crutches.

Evaluation of final outcome was done according to Thoresenet al ${ }^{7}$ classification system which includes malalignment of femur, shortening of femur, knee motion and pain/swelling. An excellent result meant that patient had full, pain-free motion of knee, malalignment and shortening was minimum.
In a good result patient had moderate pain without objective findings, 5-10 degree malalignment and $1-2 \mathrm{~cm}$ shortening. A fair result meant patient had pain that limited his recreational activities,90-120 degree knee movement, 2-3cm shortening, and 1015 degree malalignment. In poor result patient had severe pain, knee movement less than 90 degree, shortening more than $3 \mathrm{~cm}$ and malalignment more than 15 degree. Excellent and good results are considered as satisfactory and fair and poor results are considered as unsatisfactory.

\section{Results}

This prospective study of fixation of femoral shaft non-union with implant failure by SIGN interlocking intramedullary nail was carried out between January 2009 to December 2016. At first 52 cases of femoral shaft nonunion with implant failure was included in this study but due to various reasons 2 patients were not available for final follow up. So analysis of the results was done in rest of 50 cases.

Table - I

Showing age distribution of patients $(n=50)$

\begin{tabular}{|l|l|l|}
\hline $\begin{array}{l}\text { Age group } \\
\text { (yrs) }\end{array}$ & No. of patients & Percentage \\
\hline $11-20$ & 7 & $14 \%$ \\
\hline $21-30$ & 18 & $36 \%$ \\
\hline $31-40$ & 13 & $26 \%$ \\
\hline $41-50$ & 6 & $12 \%$ \\
\hline $51-60$ & 4 & $8 \%$ \\
\hline Above 60 & 2 & $4 \%$ \\
\hline
\end{tabular}

Age of the patients ranging from 11 to 70 years (mean age 34 years).Majority of the patients belongs to age group 21-40 years (62\%).

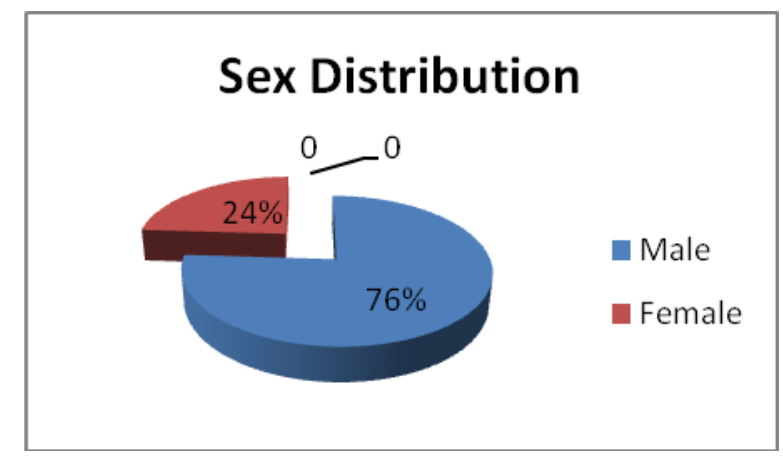


Figure-1: Pie diagram showing the sex distribution of the patients $(n=50)$

Male female ratio is 3.17:1

Table - II

Showing previous implants of patients $(n=50)$

\begin{tabular}{|l|l|l|}
\hline $\begin{array}{l}\text { Previous } \\
\text { Implants }\end{array}$ & No. of patients & Percentage \\
\hline $\begin{array}{l}\text { Interlocking } \\
\text { SIGN nail }\end{array}$ & 8 & $16 \%$ \\
\hline $\begin{array}{l}\text { Interlocking nail } \\
\text { other than SIGN }\end{array}$ & 16 & $32 \%$ \\
\hline K nail & 20 & $40 \%$ \\
\hline Plates & 6 & $12 \%$ \\
\hline
\end{tabular}

Majority of the patients (72\%) had interlocking nail other than SIGN and K-nail.

Table - III

Showing etiology of fracture of patients $(n=50)$

\begin{tabular}{|l|l|l|}
\hline Etiology & $\begin{array}{l}\text { No. of } \\
\text { patients }\end{array}$ & Percentage \\
\hline Motor vehicle & 26 & $52 \%$ \\
\hline Motor cycle & 14 & $28 \%$ \\
\hline Autopedestriain & 4 & $8 \%$ \\
\hline Fall on ground & 3 & $6 \%$ \\
\hline Other & 3 & $6 \%$ \\
\hline
\end{tabular}

Majority (88\%) of the patients had femoral shaft nonunion due to road traffic accident.

Table - IV

Showing results of the present series $(n=50)$

\begin{tabular}{|l|l|l|}
\hline Grading & No. of patients & Percentage \\
\hline Excellent & 30 & $60 \%$ \\
\hline Good & 14 & $28 \%$ \\
\hline Fair & 4 & $8 \%$ \\
\hline Poor & 2 & $4 \%$ \\
\hline
\end{tabular}

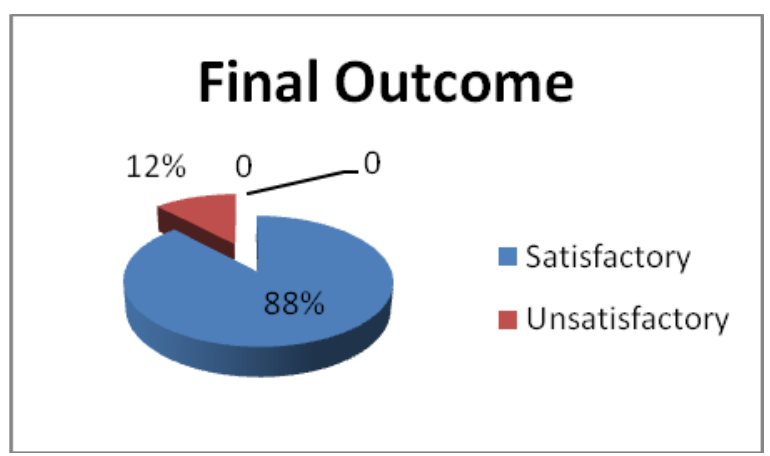

Figure-2: Pie diagram showing final outcome of the treatment of present series $(n=50)$.

In the final follow up the results were evaluated by classification system described by Thoresenet $\mathrm{al}^{7}$, 30 patients $(60 \%)$ were excellent, 14 patients (28\%) were good, 4 patients (8\%) were fair and 2 patients (4\%) were poor. At the end satisfactory result was $88 \%$ and unsatisfactory result was $12 \%$.

\section{Discussion}

A non-union is traditionally classified into either a hypertrophic or atrophic type for the convenience of treatment. The former is usually due to loss of fracture stability and the latter is due to loss of osteogenic power. The principle of treatment of former is to provide stability and the latter is increase osteogenic power. ${ }^{8}$ Variousnonoperative or operative techniques are available. However, maintenance of sufficient stability with supplementation of cancellous bone grafts has been most convincing and has achieved the highest success rate. ${ }^{3}$ According to reports in the literature, exchange nailing that provides internal bone grafts have been reported to be superior to open bone grafting. ${ }^{9,10}$ The reported success rate of exchange nailing to treat femoral shaft delayed unions or nonunion is $53-100 \% .^{9}$ Due to small incision wound for nail inlet and no exploration of the fracture site, operating time can be shortened decrease the complication rate. Factors favouring fracture healing are minimal gap, adequate stability and sufficient nutrient supply. ${ }^{8}$ If there is bone defect open bone grafting should be performed. ${ }^{11}$

The mechanism of initial injury included $88 \%$ due to high energy trauma, 6\% due to low energy 
trauma as a result of fall on the ground and 6\% due to other cause. Among the high energy trauma $59 \%$ sustained injury from motor vehicle accident, $32 \%$ from motorcycle accidents and 9\% due to autopedestrain accidents.Bredjikhan et $\mathrm{al}^{1}$ andSabur et $\mathrm{al}^{12}$ reportedin their study $65 \%$ and $80 \%$ respectively were due to high energy trauma.

In this study 32(64\%) nonunion involved the right femur and 18(36\%) involved the left femur. Saburet $\mathrm{al}^{12}$ reported in his study $15(60 \%)$ nonunion involved the right femur and $10(40 \%)$ involved the left femur.

Involvement of proximal third of the femur 30\% $(\mathrm{n}=15)$, middle third $50 \%(\mathrm{n}=25)$ and distal third $20 \%(n=10)$. Saburet $a^{12}$ reported in his study involvement of proximal third of the femur 20\% $(n=5)$, middle third 50\% $(n=13)$ and distal third $30 \%(\mathrm{n}=7)$.

Initial treatment of the femoral shaft fracture includedInterlocking SIGN nail $16 \%(\mathrm{n}=8)$, Interlocking nail other than SIGN nail 32\% $(n=16)$, Kuntscher nail $40 \%(n=20)$, Plates $12 \%$ $(\mathrm{n}=6)$. Saburet $\mathrm{al}^{12}$ reported in his study interlocking Intramedullary nail $40 \%(n=10)$, Kuntscher nail 40\%(n=10), DCP 16\% $(n=4)$ and Kuntscher nail augmented by DCP 4\% (n=1).

In this study the average follow up of the patient was 20 months (range 12-24 months). In Saburet $\mathrm{al}^{12}$ it was 16 months (range 14-22 months).

Closed exchange nailing was done in 28 cases (56\%) and open nailing was done in 22 cases (44\%). Open methods were done due to broken SIGN nail in 3 cases (13.6\%), broken other interlocking nail in 7 cases (31.8\%), broken K-nail in 6 cases (27.2\%) and plates in 6 cases (27.2\%). Reaming materials were used asinternal bone graft in all the cases. Autogenouscancellous bone graft was given in 5 cases due to comminution at fracture site(2 cases) and severe osteoporosis (3 cases).Sabur et $\mathrm{al}^{12}$ reported in his study of using only one patient needed iliac crest bone graft due to more comminution at the fracture site in only one patient.

Most of the nonunion were treated 1-2 years after the the initial ijury. 2 patients developed superficial infection and 2 patients develop deep infection. Out of 50 patients, 48 patients were united, 2 patients developed delayed union but went on healing. $20 \%$ patients had $2-3 \mathrm{~cm}$ femoral shortening, $20 \%$ patients had deformity, 20\% patient had restricted knee movement. 2 patients developed superficial wound infection, which were improved by regular wound dressing, 1 patient developed deep wound infection, which was improved by repeated wound debribement. In all the 3 cases proper antibiotics were used as per culture and sensitivity reports.

In the final follow up the results were evaluated by classification system described by Thoresenet $\mathrm{al}^{7}$, the satisfactory result (excellent and good) of this series was $88 \%$ and unsatisfactory result (fair and poor) was $12 \%$.In Saburet al ${ }^{12}$, it was $84 \%$ and $16 \%$ respectively.

\section{Conclusion}

SIGN interlocking nailing can be done without Carm and it is donated by SIGN fracture care internationals. Long term follow up result of using SIGN nail in the treatment of femoral shaft nonunion with implant failure is very satisfactory.

\section{References}

1. Beredjiklian PK, Naranja RJ, Heppenstall RB, Brighton CT, EsterhaiJL.Results of treatment of 111 patients with nonunion of femoral shaft fractures.The University of Pennsylvania Orthopaedic Journal.1999; 12: 52-56.

2. Kempf I, Grosse A, Beck GF. Closed locked intramedullary nailing: its application to comminuted fractures of the femur.J Bone Joint Surg [Am].1985; 67: 709-20.

3. Brav EA. The use of intramedullary nailing for nonunion of the femur.ClinOrthop.1968; 60: 69-75.

4. Oh I, Nahigian SH, Rascher JJ, Farrall JP. Closed intramedullary nailing for ununited femoral shaft fractures. ClinOrthop.1975; 106: 206-15.

5. Chao EYS, Inoue N, Ellias JJ, Aro H. Enhancement of fracture healing by mechanical and surgical intervention. ClinOrthop. 1998; 355S: 163-78.

6. Winquist RA, Hansen ST, Clawson DK. Closed intramedullary nailing femoral fractures. J Bone Joint Surg [Am].1984; 66: 529-39.

7. Thoresen RH, Web LX, Winquist RA, Hansen ST. Intramedullary nailing and reaming for delayed union of the femoral shaft. ClinOrthop.1986; 212: 133-41. 
8. Wu CC. The effect of dynamization on slowing the healing of femoral shaft fractures after interlocking nailing. J Trauma. 1997; 43: 263-7.

9. Wu CC, Chen WJ. Treatment of femoral shaft aseptic nonunions: comparison between closed and open bone grafting techniques. J Trauma. 1997; 43: $112-6$.

10. Chapman MW. The effect of reamed and undreamed intramedullary nailing on fracture healing.ClinOrthop. 1998; 355S: 230-8.
11. Wolinsky P, Tejwani N, Richmond JH, Koval KJ, Egol K, Stephen DJG. Controversies in intramedullary nailing of femoral shaft fractures.J Bone Joint Surg [Am].2001; 83: 1404-15.

12. Sabur MA, Saleheen MS, Maleque MA, Pathan $M A H$, Sarwar MG, Qavi MI. Results of fixation of femoral shaft non-union with implant failure by SIGN interlocking nail. J Bangladesh Orthop Soc. July 2012; 27(2):143-8. 